\title{
Biotechnological bases of the global ecological crisis
}

\author{
Valery Galynkin ${ }^{1, *}$ and Alfiya Gabidova ${ }^{2}$ \\ ${ }^{1}$ Saint Petersburg State Institute of Technology (Technical University), 190013, 26 Moskovsky \\ prospect, St. Petersburg, Russia \\ ${ }^{2}$ Federal State Unitary Enterprise "Moscow Endocrine Plant”, 25, Novokhokhlovskaya Str., 109052, \\ Moscow, Russia
}

\begin{abstract}
Anthropogenic impacts on nature endangered the normal implementation of its inherent biotic processes, upset the balance of the state of the biosphere. The most important function of any biocenosis, biogeocenosis and biosphere is the regular recreation of living matter and the energy accumulated in it. Meanwhile, it is the soil cover that ultimately takes on the pressure of the flow of industrial and municipal emissions and waste, performing the most important role of a buffer and detoxifier. The soil accumulates heavy metals, pesticides, hydrocarbons, detergents and other chemical pollutants, thereby preventing their entry into natural waters and purifying the atmospheric air from them. On the other hand, the same substances or their derivatives, as well as heavy metals, fluorine, nitrogen and sulfur oxides in their original or transformed form, are intensively bound by mineral and organic substances of the soil, which sharply reduces their availability to plants and, accordingly, the general level of toxicity. Zinc content in soil ranges from 10 to $800 \mathrm{mg} / \mathrm{kg}$, although most often it is $30-50 \mathrm{mg} / \mathrm{kg}$. The greatest buffer capacity and the ability to reduce the negative impact of pollutants on plant and animal organisms are found in soils with a high humus content, with a heavy particle size distribution, high absorption capacity, enriched with lime materials (carbonates).
\end{abstract}

\section{Introduction}

For the development of the material sphere, modern civilization, formed within the biosphere, has created, and continues to create technologies that destroy it [1]. Even environmental technologies imply damage to a part of the biosphere and the withdrawal of any resource. A period of post-industrial integral world civilization is coming. Based on the development and dissemination of the postindustrial technological mode of production, it will be possible to ensure high rates of labor productivity, overcoming the growth of depletion of natural and labor resources. The biosphere has a colossal self-cleaning ability, but these abilities of nature are not unlimited [2].

Anthropogenic impacts on nature endangered the normal implementation of its inherent biotic processes, upset the balance of the state of the biosphere. The anthropogenic load on

\footnotetext{
${ }^{*}$ Corresponding author: 7731254@mail.ru
} 
the natural environment has reached such proportions today that it has led to a global ecological crisis. The destructive activity of man gave rise to a conflict between society and nature, created risks that are called environmental [3]. The most important function of any biocenosis, biogeocenosis and biosphere is the regular recreation of living matter and the energy accumulated in it. If in the field of control and protection of atmospheric air and natural waters there are certain achievements, including the creation of a network of special laboratories, methods of analysis and MPCs for a fairly large number of substances and elements have been developed, then in the field of monitoring and protection of soils, progress is still insignificant. Meanwhile, it is the soil cover that ultimately takes on the pressure of the flow of industrial and municipal emissions and waste, performing the most important role of a buffer and detoxifier. The soil accumulates heavy metals, pesticides, hydrocarbons, detergents and other chemical pollutants, thereby preventing their entry into natural waters and purifying the atmospheric air from them. In the soil, many chemical pollutants undergo profound changes. Hydrocarbons, pesticides, detergents and other compounds can be mineralized or transformed into substances that do not have a toxic effect on soil, microorganisms, plants, animals and humans.

\section{Soil protection}

Protection of soils, their rational use are of paramount importance for the economic and social development of the country. The significance of the current state of soil resources, their rational use, and respect for them will serve to increase their fertility. In the second half of the twentieth century, the volumes and rates of technogenic pollution of the environment increased so much that it took the adoption of special international programs for the protection of nature. In 1972, the UN Environment Program was developed, including the problems of monitoring the natural environment for the purpose of early warning of impending natural or anthropogenic changes that may harm human health or well-being. Ecological well-being or the quality of the natural environment surrounding a person, which, in relation to a person, does not lend itself to a clear definition.

On the other hand, the same substances or their derivatives, as well as heavy metals, fluorine, nitrogen and sulfur oxides in their original or transformed form, are intensively bound by mineral and organic substances of the soil, which sharply reduces their availability to plants and, accordingly, the general level of toxicity. Zinc content in soil ranges from 10 to $800 \mathrm{mg} / \mathrm{kg}$, although most often it is $30-50 \mathrm{mg} / \mathrm{kg}$. The accumulation of an excess amount of zinc adversely affects most soil processes: it causes a change in the physical and physicochemical properties of the soil, and reduces biological activity. Zinc inhibits the vital activity of microorganisms, as a result of which the processes of formation of organic matter in soils are disrupted. An excess of zinc in the soil makes it difficult for the fermentation of cellulose decomposition, respiration, and the action of urease.

The greatest buffer capacity and the ability to reduce the negative impact of pollutants on plant and animal organisms are found in soils with a high humus content, with a heavy particle size distribution, high absorption capacity, enriched with lime materials (carbonates). These soils include the most fertile chernozems, some rendzins, and floodplain lands. This gives the soil a natural resistance to the effects of chemical pollutants and allows you to get high and high-quality full-value yields of the most important agricultural crops, even in industrialized regions.

In the CIS, a nationwide system of observation and control over the state and level of environmental pollution has been created.

Unfortunately, the natural resistance of soils, their natural buffering capacity are not unlimited. According to the highlights of B.G. Rozanov and other scientists, about two billion hectares of agricultural soil were lost in the world for various reasons. Land losses 
caused by irrigation alone have amounted to about one hundred million hectares over the past three hundred years, and about the same area is now occupied by soils with reduced productivity due to salinization. The loss of humus is very high, on which practically all the most important properties of soils and their resistance to unfavorable situations depend. Apparently, during the period of agricultural culture, the soil cover has lost up to $15 \%$ of the original supply of organic matter. Moreover, these negative phenomena have been proceeding especially rapidly in recent decades.

Plant nutrition from the first days of its existence was carried out by underground structures (rhizoids, rhizomes) that come into contact with soil substrates. The evolution of the plant world led to the emergence of a symbiosis of plants and soil microorganisms. This limitation was compensated by the formation of arbuscular mycorrhiza (AM), a symbiosis of plants with glomus fungi (Glomeromycota), which have a high osmotrophic activity.

Plants are characterized by the most profound dependence on the functions provided by microorganisms. The deficiency can be compensated for by plant-microbial symbiosis due to the fact that plants, possessing a high activity of photosynthesis, must create an excess of C-compounds, which are produced by microorganelles of the plant cell and provide them with fungi and bacteria, which serve as donors for the host of missing nitrogen, phosphorus and mineral components ... They enter microorganisms from the soil and are transferred in a convenient form to plants. The nutrition of the most ancient plants turned out to be underground organs (rhizoids, rhizomes) in contact with soil substrates, however, due to insufficient adsorption activity, these organs could not fully cope with the provision of the entire plant organism with mineral substances and water. Therefore, the underground organs of plants from the very beginning of their evolution performed, along with the mechanical function (fixation in the substrate), also symbiotic functions, including the placement of symbiotic microbes and metabolic integration with them. When trophic symbiosis occurs, the mutual transitions of pathogens and mutualists are much more limited: even if the relationship between them can be traced, as in the case of rhizobia and agrobacteria, the gene systems of symbiosis turn out to be fundamentally different. A widespread method for the emergence of useful plant symbionts can be considered to be their "recruitment" from among the companions of numerous organisms interacting with plants, for example, from among bacteria that are antagonists or mutualists of mycorrhizal, endophytic or pathogenic fungi, as well as phytophagous insects.

Due to unsatisfactory sanitary and hygienic and epidemiological conditions in many populated areas, in everyday life, in medical and preventive institutions (LPI), at various public utilities, etc., as well as in connection with the difficult socio-economic situation in our country, the level morbidity of the population with tuberculosis, meningitis, viral hepatitis, salmonellosis, dysentery and other intestinal infections, acute respiratory diseases, fungal infections, etc. continues to remain high.

\section{Biofilms}

Biofilms are highly organized, mobile, continuously changing heterogeneous communities, consisting of both actively functioning cells and dormant forms enclosed in an exopolymer matrix. They can consist of one [1] or, more often, of several types of microorganisms]. Previously, it was believed that biofilms are formed only on medical devices, such as catheters, endotracheal tubes, intrauterine devices, contact lenses [2]. It has now been established that biofilms are the main factors in the pathogenesis of diseases characterized by chronic inflammation [1,]. They are found in more than $80 \%$ of chronic infectious and inflammatory diseases, which made it possible to put forward the concept of chronic diseases as diseases of biofilms $[2,3]$. 
Biofilm formation is a complex complex dynamic process consisting of several stages: adhesion of cells to the surface and redistribution of cell mass; active cell division to create cell clusters; the formation of an exopolymer mucous matrix. The initial attachment of a microbial cell to the substrate surface is carried out due to the action of electrostatic, hydrophobic forces, van der Waals forces, and nonspecific adhesion. Adhesion to biological surfaces is due to the specific interaction of adhesin proteins or lectins of the fimbriae of the exoplasmic compartment of a bacterial cell with receptors or specific domains of the target cell membrane surface. The mechanism of adhesion of gram-positive bacteria is different from that of gram-negative bacteria. Biofilms can be characterized as bacteria embedded in a thick mucous layer made of sugars and proteins. This film barrier protects microorganisms from external influences [2]. Microorganisms that make up the biofilm exist in two forms: fixed to the surface and planktonic, floating, which is the substrate for the spread of infection from its primary locus]. The ability of bacteria to form biofilms is an essential factor in pathogenicity. Biofilms are physical structures with unique characteristics formed by microbial communities associated with surfaces. Plant-microbial symbiosis (PMC) is attracting increasing attention of researchers, plants are characterized by the deepest dependence on the functions provided by microorganisms since plants. many of the metabolic capabilities and behaviors required for independent existence. Their deficit can be replenished due to the fact that plants, possessing a high activity of photosynthesis, must create an excess of C-compounds and "share" it with fungi and bacteria, which serve as donors for the host of missing ecologically sign functions. A significant number of microorganisms in natural and artificially created environments exist in the form of structured communities attached to the surface - biofilms. Biofilm is a microbial grouping characterized by cells attached to the surface or to each other, which are in the matrix of extracellular polymeric substances synthesized by them. They demonstrate a change in the phenotype, expressed by changes in growth parameters and the expression of specific genes. The film usually includes $15-20 \%$ of the bacterial mass, which is firmly attached to a particular surface, and $80-85 \%$ of the protective matrix, which reduces the effect of antibiotics and antiseptics on target microcultures by tens, hundreds and even thousands of times [1]. Biofilms are mobile, continuously changing heterogeneous groupings that can consist of one species of bacteria or fungi, or, more often, can be heteromicrobial (i.e., contain many different types of microorganisms). Biofilms can be characterized as bacteria embedded in a thick mucous layer made of sugars and proteins. This film barrier protects microorganisms from external influences [2]. Microorganisms that make up the biofilm exist in two forms: fixed to the surface and planktonic, free-floating, which is the substrate for the spread of infection from its primary locus. Biofilm formation is one of the main strategies that enhances the survival of bacteria in the environment, including in the host. The ability of microorganisms to exist as part of biofilms creates great difficulties, because this significantly increases the resistance of bacteria to antibacterial and disinfectants, the influence of unfavorable environmental factors (such as low or high values of $\mathrm{pH}$, high osmotic force and other factors, as well as the actions of the immune defense of host organism).

The growth potential of any bacterial biofilm is limited by the amount of nutrients in the environment, their availability to the cells contained within the biofilm, and the ability to remove metabolic products. In addition, there is a hydrodynamic optimum of the flow rate of the environment, which accelerates the growth of biofilm by optimizing the rate of supply of nutrients and removal of metabolic products, and in the case of a higher rate, it causes erosion of the outer layers of the biofilm. After the final maturation in the biofilm, the optimal growth rate and cell death, physiological and metabolic efficiency are established, which provide optimal conditions for functional coordination, as a result of which a three-dimensional biofilm structure is created - non-structurally homogeneous 
microbial cells on the surface. Rather, they can be described as heterogeneous structures in time and space, but the basic structure of the community is universal, with some minor variations. Once stably attached, bacteria begin to form a surrounding matrix known as an extracellular polymeric substance. It is a matrix measure or "slime" (EPS-matrix) [2]. This matrix consists of a mixture of exopolysaccharides released into the environment (), proteins, nucleic acids, and other substances. Bacterial exopolysaccharides are the main component of the biofilm matrix. Its main component is bound water [3]. Like the intercellular matrix of animal tissues, the microbial matrix also includes fibrillar elements. The similarity between animal and microbial matrix is complemented by the commonality of some chemical components (for example, sialic acids. All biofilms are hydrated, some up to $73 \%$ of extracellular material, including water channels and exopolysaccharides. In most species, the matrix consists of alginate, being predominantly anionic. Matrix - a threedimensional structure that surrounds, anchors and protects microcolonies of bacteria attached to various surfaces [4]. The matrix is divided by channels filled with water and also has cavities. Through the channels, nutrients are transported and convective flows of oxygen pass from the outer to the inner parts of the biofilm, while metabolites of bacterial cells are eliminated. The pores and channels that permeate the entire biofilm are a very important part of its structure.

\begin{tabular}{lll}
\hline Planktonic, freely moving forms $\quad$ Substrate adhesion & $\begin{array}{l}\text { Irreversible adhesion, } \\
\text { intense cell division, } \\
\text { matrix formation }\end{array}$ & Formed biofilms
\end{tabular}

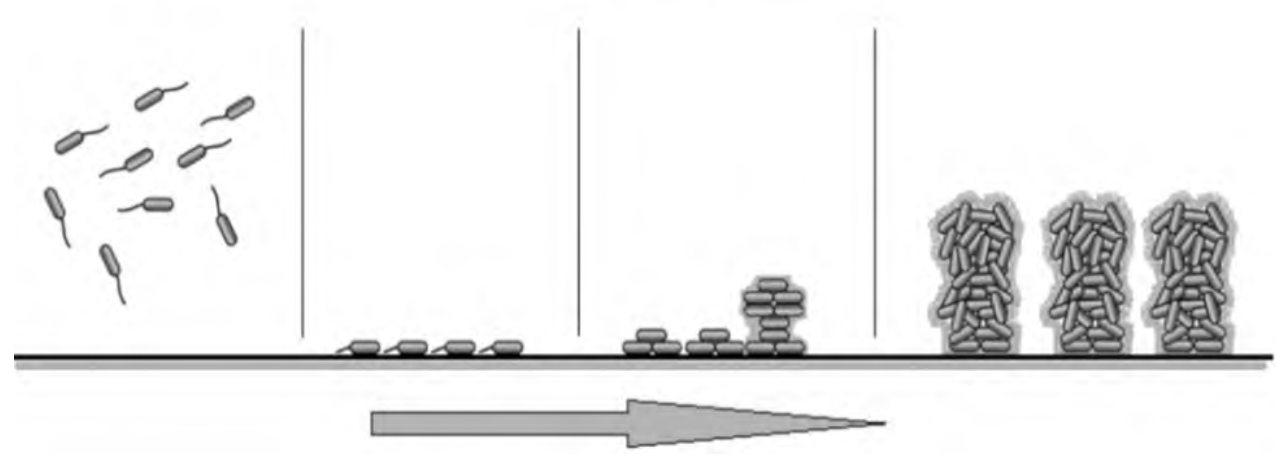

Fig. 1. Diagram of the process of formation of bacterial biofilms.

Soil as a habitat for organisms is a heterogeneous three-phase system, including soil air, soil moisture, and mineral particles. As a result of the activity of living organisms, dead organic matter - humus - and a live mass of roots and microorganisms are added to the soil. The heterogeneity of the soil is due to the presence of particles of different sizes, and this hierarchy of sizes includes: Macroaggregate $\rightarrow 2000 \mu \mathrm{m}$, microaggregate (sand) (decomposable organic matter) - $200 \mu \mathrm{m}$, root hairs (resistant organic matter) - $20 \mu \mathrm{m}$, large pores (stable organic matter) - $2 \mu \mathrm{m}$, pores ( stable mineral part) -0.2 microns. The pore space of the soil of the order of microns, filled with water and soil air, is available for the development of microorganisms. Microorganisms occupy less than 0.01 pore space. The position within the pores protects microorganisms from predators to a certain extent. Micropores less than 0.2 microns, for example a cover made of clay minerals, physically protect organic matter from the effects of exoenzymes. The formation of aggregates occurs with the participation of microorganisms. It is due to the sticking effect of bacterial mucus on small mineral components of the soil, primarily clay particles. Microscopic analysis of the aggregates in their central part reveals lumps of bacteria, and outside the covering mycelium felt. Bacteria penetrate into the pore space of the unit with pores of at least 0.6 
microns, if the pores are not occupied by soil moisture. The inside of the unit remains moist, while the surface is dried and in equilibrium with the moisture of the soil air. The soil aggregate turns out to be the habitat of the microbial community, created with the participation of functionally different groups of microorganisms interacting in it. Soil moisture, including film moisture, is the habitat of bacteria and partly fungi, the mycelium of which can enter the gas phase. Soil water has a matrix potential, which depends on its binding to the surface of particles, and an osmotic potential, which depends on dissolved substances. The pore space of the soil depends on its nature and is usually $50-60 \%$ of its volume, oxidizer biota, which is in dynamic equilibrium with the atmospheric layer in the vegetation cover (aerotope). The water in the soil is part of the soil solution, which serves as a habitat for bacteria. Composition of the soil solution Soil moisture, including film moisture, is a habitat for bacteria and partly fungi, the mycelium of which can enter the gas phase. The most interesting is the association of microorganisms with the root system of living plants. The roots are located in the soil horizon, the richest in organic matter (Fig. 3).

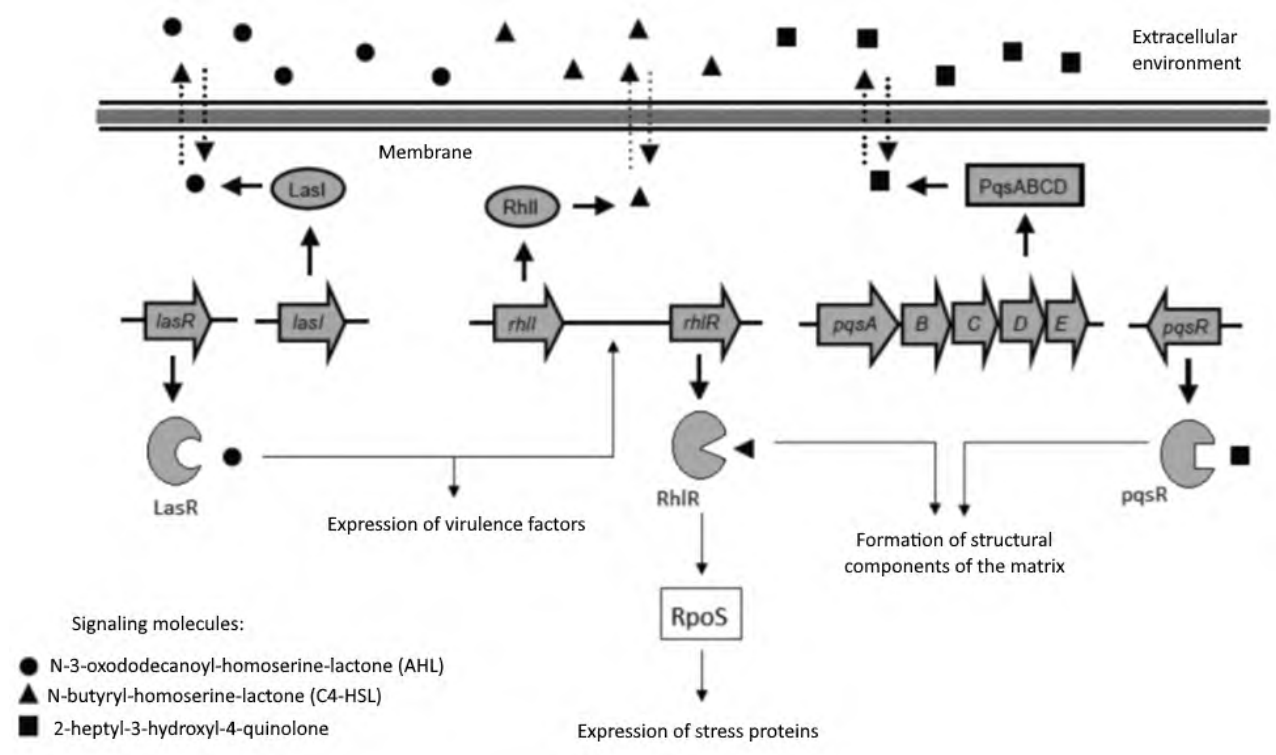

Fig. 2. Scheme "Quorum sensing" in P. aeruginosa, three signaling systems are presented.

Interaction with the root system includes three areas: 1) the area of the soil with the direct impact of the roots - rhizosphere; 2) the surface of the root - rhizoplane; 3) root tissue. The variety of microorganisms near the root is determined by the variety of incoming substances, the variety of trophic interactions of microorganisms with each other (hydrolytics and dissipotrophs, bacteriolytic loop) and the effect of specific plant substances. A very wide range of organotrophic aerobic organisms can be expected here, the nutritional requirements of which are focused on exudates. As a rule, this area of development of microorganisms is characterized by an excess of organic carbon and a limiting content of nitrogen and phosphorus. 


\section{Phylosphere \\ Epiphytic microorganisms}

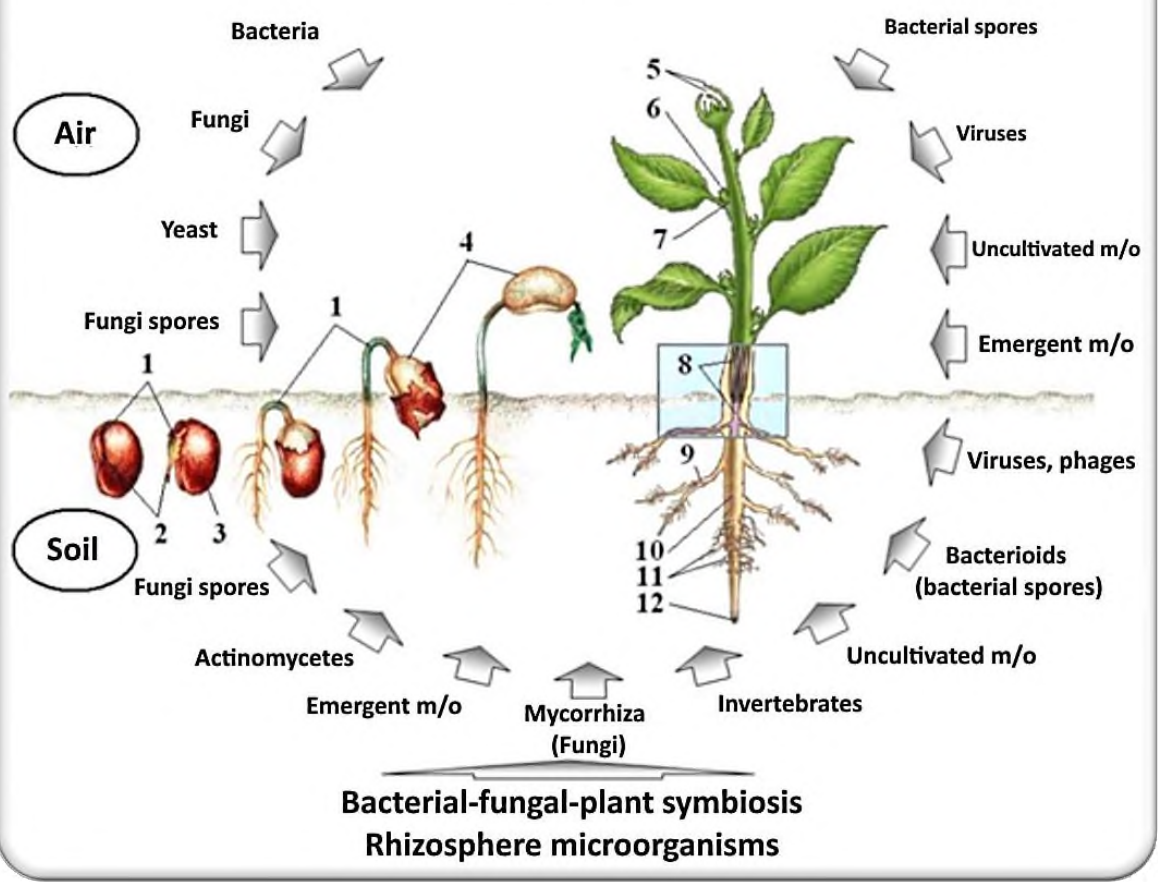

Fig. 3. Interaction of a plant with the environment.

\section{References}

1. V.I. Vernadsky, The biosphere. Springer Science \& Business Media (1998)

2. V. Gorshkov, Physical And Biological Foundations Of Sustainability Of Life (Moscow, VINITI, 1995)

3. A.E. Gabidova, V.A. Galynkin, Biological bases of resistance. RAE RF (2018)

4. I.B. Pavlova, A.N. Antonova, E.M. Lenchenko, Study of biofilm formation by pathogenic bacteria, Russian journal Problems of veterinary sanitation, hygiene and ecology (2), 63-70 (2016)

5. V.V. Gostev, S.V. Sidorenko, Bacterial biofilms and infections, Journal of Infectology 2 (3), 4-15 (2014)

6. S.Singh, I. Chowdhury, R. Singh, Understanding the mechanism of bacterial biofilms resistance to antimicrobial agents, The open microbiology journal 11, 53 (2017)

7. J. Yan, B.L. Bassler, Surviving as a community: antibiotic tolerance and persistence in bacterial biofilms, Cell host \& microbe 26(1), 15-21 (2019)

8. C.A. Phillips, Bacterial biofilms in food processing environments: a review of recent developments in chemical and biological control, International Journal of Food Science \& Technology 51(8), 1731-1743 (2016) 